\title{
GAIA Level 2 Intrapartum Stillbirth
}

National Cancer Institute

\section{Source}

National Cancer Institute. GAIA Level 2 Intrapartum Stillbirth. NCI Thesaurus. Code C128023.

GAIA Level 2 Intrapartum Stillbirth is defined by four criteria: first, the delivery of an infant with no signs of life at birth, including the following: no spontaneous movements, no umbilical cord pulse, no heartbeat, no respirations, and an Apgar score of 0 at both 1 and 5 minutes. Determination of the absence of signs of life is made by physical examination after delivery, with or without electronic monitoring of heart rate, respiratory rate, and pulse oximetry OR documentation of lack of response to resuscitation efforts; second, evidence of a live fetus prior to the onset of labor (maternal report of fetal movement prior to the onset of labor and documentation of fetal heart tones by auscultation or hand held Doppler). NOTE: In the absence of evidence of a live fetus prior to the onset of labor, the fetal death should be reported as a stillbirth or an antepartum stillbirth; third, attended delivery followed by post-delivery physical examination that is performed by a health care professional who meets the standard of care in the immediate health care setting, and which is consistent with intrapartum death; fourth, gestational age within the pre-defined range for selected stillbirth definition as assessed by maternal and/or fetal parameters (level 1-2 in GA assessment algorithm). 